\title{
APROXIMACIÓN A LA POSIBLE EXISTENCIA DE LA SEÑAL DE LOS CICLOS SOLARES 18 Y 19 EN LA PLUVIOMETRÍA DE LA PENÍNSULA IBÉRICA*
}

\author{
Elisabet Romeu i Codina' y Joan-Albert López-Bustín ${ }^{2}$
}

\begin{abstract}
Resumen: A partir de la metodología empleada en un estudio de los E.E.U.U. (Perry, 1994), se hallan correlaciones significativas entre la pluviometría ibérica y el período solar más activo registrado (1939-1969), con un desfase de tres años, para el ámbito atlántico del norte peninsular, y de 4 años, para el del suroeste. Estos retrasos corresponden al período de desplazamiento, desde las calmas tropicales noratlánticas, de anomalías térmicas marinas en el seno de la corriente del Golfo. No obstante, el resultado más inesperado es la alta correlación hallada con la pluviometría del golfo de Valencia sin desfase temporal, hecho atribuible a la configuración reducida y semicerrada del mar Mediterráneo.
\end{abstract}

Palabras clave: Ciclo solar de Schwabe. Pluviometría de la Península Ibérica. Coeficiente de correlación de Pearson. Corriente del Golfo. Anomalía de la temperatura superficial del mar (Tsm).

\begin{abstract}
Based on the methodology used in a study in the U.S. (Perry, 1994), significant correlations are found between rainfall in the Iberian Peninsula and the most active solar period registered (1939-1969), with a three-year lag for the Atlantic area in the north of the Iberian peninsula, and a four-year lag for the southwest. These delays correspond to the shifting period of sea thermal anomalies within the Gulf current from the north Atlantic tropical calm. Nevertheless, the most unexpected result is the high correlation found in rainfalls on the gulf of Valencia with no temporal gap, a fact that is attributable to the reduced and semi-closed configuration of the Mediterranean.
\end{abstract}

Key words: Schwabe's solar cycle. Rainfall on the Iberian Peninsula. Pearson's correlation coefficient. Gulf current. Sea surface temperature (SST) anomaly.

\footnotetext{
* Recibido: 11-4-2005. Aceptado: 28-2-2006.

1 Facultat de Física i Química de la Universitat de Barcelona.

2 Grup de Climatologia de la Universitat de Barcelona. Departament de Geografia Física i AGR. Facultat de $\mathrm{G}^{\mathrm{a}}$ i $\mathrm{H}^{\mathrm{a}}$. C/ Baldiri i Reixac s/n, 08028 Barcelona (jlopezbustins@ub.edu)
} 


\section{Introducción}

\subsection{Síntesis bibliográfica}

En el contexto del "Cambio Climático" se tiende a infravalorar el papel desempenado por la actividad solar y su variabilidad; así es como yace subyugado a otros factores en la elaboración del pasado tercer IPCC (Intergovernmental Panel on Climate Change). Sin embargo, se han encadenado recientemente unas líneas de investigación paralelas vinculadas a esta variabilidad de la actividad solar. Es bien conocido entre el mundo científico que esta actividad fluctúa de forma cíclica, y, en consecuencia, se pretende hallar oscilaciones similares en variables climáticas terrestres. Esta simple relación ha sido tradicionalmente establecida por múltiples estudios en el siglo XIX y $\mathrm{XX}$, recopilados en su mayoría en el trabajo "The Role of the Sun in Climatic Change" de Hoyt y Schatten de 1997. No obstante, a lo largo de las últimas décadas han surgido diversos detractores de esta relación lineal hallada con simples coeficientes de correlación y análisis espectrales, que se limitan a la búsqueda de un ciclo, desprovistos de una relación científica (Burroughs, 1992; Vázquez Abeledo, 1998). Nuevas metodologías de vanguardia han puesto de manifiesto la imbricación de múltiples variables en la modulación de la señal solar. Cabe ejemplificarlo con el reciente trabajo llevado a cabo por Labitzke y van Loon (1999) en el estudio de la influencia solar en la estratosfera y su complejo modo de transmisión a la troposfera (Baldwin y Dunkerton, 1999 y 2005). En consecuencia, se supera la tradicional relación lineal tras detectar unas pulsaciones irregulares en el tiempo y en el espacio de la señal solar.

En el ámbito internacional se han seguido distintas líneas de investigación en la búsqueda de las diversas vías de la hipotética incisión del sol en el clima terrestre. Incluso ciertas teorías, entre las más radicales, anuncian drásticos cambios climáticos para mediados del siglo XXI mediante cálculos de predicción de la oscilación de determinados ciclos solares más extensos, de 83 años, ciclos de Gleissberg (Landscheidt, 1995 y 2000). Sin embargo, en España también se han consolidado líneas de investigación propias en la temática. Son muestra de ello el trabajo realizado por Vaquero (2002 y 2004) de la Universidad de Extremadura; en particular, su reciente aportación, que vincula las inundaciones del Tajo durante el pasado milenio (Benito et al. 2003) con el comportamiento de la NAO modulado en función de la actividad solar (Kirov y Georgieva, 2002). Asimismo, en esta misma línea de estudio, se hace hincapié en el trabajo de Montón Chiva y Quereda Sala (1997) para el ámbito mediterráneo peninsular. En una vía distinta, que intenta relacionar la nubosidad con la fluctuación de la cantidad de los rayos cósmicos que alcanzan la superficie terrestre en función de la expansión -contracción de la beliosfera- se hallan las investigaciones de Pallé et al. (2004), y Lucio et al. (2005) en Portugal. 


\subsection{Las manchas solares}

Desde 1978 las mediciones realizadas con satélites han demostrado que el total de irradiancia solar tiene un margen de variación entre 0.1 y $0.2 \%$ aproximadamente a lo largo de los 11 años del ciclo solar (Willson y Hudson, 1988).

Las manchas contribuyen a la irradiancia total haciendo disminuir la radiación de salida, pero durante estos períodos de gran actividad, en que la superficie solar está llena de estas manchas más oscuras, hay proporcionalmente un número más grande de regiones hiperactivas más brillantes que preceden y sobreviven la evolución de las manchas. Éstas zonas reciben el nombre de fáculas, las cuales tienen un efecto contrario. El balance neto de los dos fenómenos es un aumento de la irradiancia total emitida.

\subsection{Interacción de la actividad solar-océano-atmósfera}

La mitad de la radiación solar que alcanza la superficie terrestre se concentra en el espectro visible (Liov, 1980). Según Perry (1992 y 1994) existe una conexión entre esta actividad solar y la interfaz océano-atmósfera, probada en sus investigaciones para el Pacífico Norte en las variaciones de las medias anuales de la precipitación del noroeste de los Estados Unidos. El mecanismo hipotético se explica a lo largo de tres fases que retroalimentan la reducida variación solar: Una primera fase se basa en la penetración de la energía solar hasta unos determinados estratos marinos, siendo en la banda del visible donde la energía solar alcanza mayor profundidad, hasta unos 100 metros, y, sobre todo, en esas latitudes tropicales donde las aguas se encuentran más calmadas y transparentes, lo que facilita dicha penetración (Lewis et al., 1990); se propició, en consecuencia, el inicio de una anomalía térmica en las aguas marinas subsuperficiales por una mayor o menor absorción y acumulación energética. Según Reid (1991 y 2000) existe una alta correlación entre la temperatura superficial del mar (Tsm) global y la variación de la constante solar. Posteriormente, la segunda fase tiene lugar cuando estas anomalías son transportadas a otras latitudes mediante las grandes corrientes oceánicas, alcanzando zonas continentales de latitudes medias pasados unos años. Según Wallace et al. (1990), se producen ciertas anomalías en los campos de presión troposféricos en función de la variabilidad de la Tsm. En caso de una anomalía positiva, se libera la energía acumulada mediante importantes evaporaciones que aportan altos contenidos de humedad a la baja atmósfera, que, por la relativa baja densidad del aire correspondiente, favorece la formación de bajas presiones, o el refuerzo de depresiones dinámicas como la baja de las Aleutianas (Pacífico Norte) o la de Islandia (Atlántico Norte). Ello conlleva, finalmente, un aumento pluviométrico en las franjas costeras de los continentes (Figura 1). Y viceversa, una anomalía térmica negativa favorece altas presiones, que propician escasez de precipitaciones; mecanisṃno básico que probó la severa sequía del Norte de América en 1988 (Palmer 


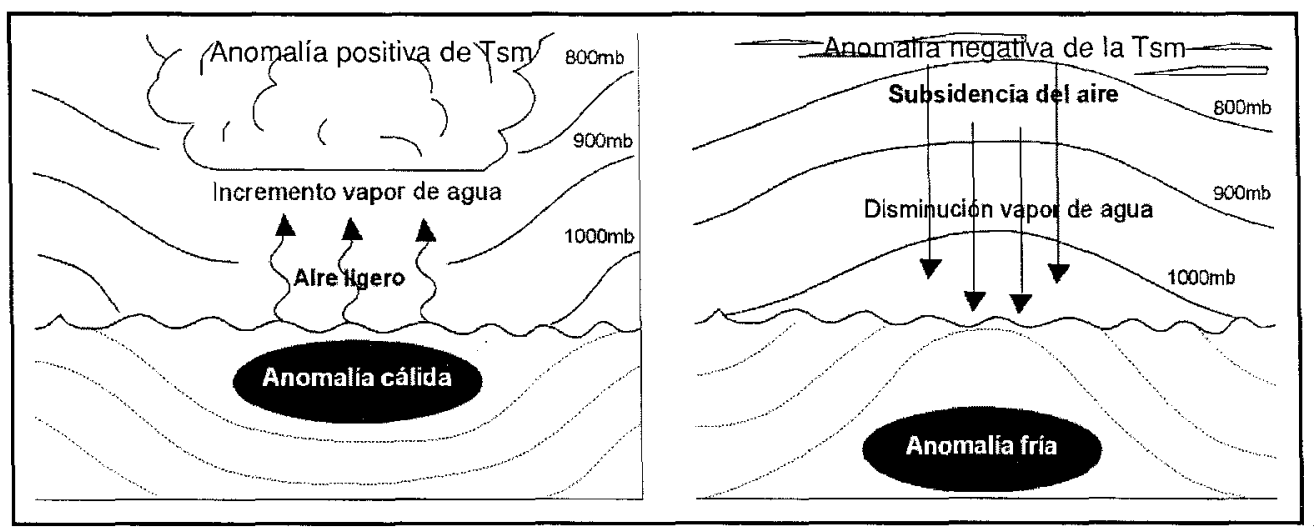

Figura 1. Esquema de la fase de transferencia energética en la interfaz océano-atmósfera.

and Brankovic, 1989). Con esta transferencia positiva o negativa de energía del océano a la atmósfera se concluyen las tres fases.

Las anomalías térmicas marinas formadas en el Pacífico Norte Tropical son comparables con aquéllas que se podrían originar en el océano Atlántico Norte en las tranquilas aguas tropicales del mar de los Sargazos que bañan las islas Bermudas, en las "latitudes de los caballos". El transporte de las anomalías térmicas superficiales marinas en el estudio de Perry (1994) es mediante la circulación horaria del océano del Pacífico Norte, siendo la velocidad de transporte entre $5-8 \mathrm{~km} /$ día, hecho que explica que la respuesta pluviométrica a las variaciones de la irradiancia del Sol se presenten al cabo de 4 años en la costa oeste de los Estados Unidos. En el Atlántico Norte, estas aguas anómalamente frías o cálidas también cabe hallarlas en las costas occidentales de Europa, relativamente más tempranas que en la costa oeste de Estados Unidos, al cabo de 2 ó 3 años, por la velocidad media de $6-8 \mathrm{~km} /$ día que caracteriza la corriente del Golfo en su deriva noratlántica, y por una menor distancia intercontinental. La corriente del Golfo se divide en tres ramales a las puertas de las aguas europeas, uno hacia las costas occidentales islandesas (corriente Irminger), un segundo ramal deriva hacia las tierras europeas septentrionales, y un último se dirige a la Península Ibérica, penetrando un brazo en el mar Cantábrico, recorriendo la costa ibérica oeste de norte a sur. Una vez alcanza el golfo de Cádiz, alimenta, en parte, al mar Mediterráneo por el estrecho de Gibraltar. Seguidamente, deriva en una corriente fría en dirección a las Islas Canarias para retornar de nuevo al continente americano (Figura 2). De este modo, se completa el giro circulatorio horario de las aguas del Atlántico Norte, regido, en todo momento, por la dinámica del anticiclón de las Azores, centro de acción equiparable al anticiclón subtropical del Pacífico Norte. 


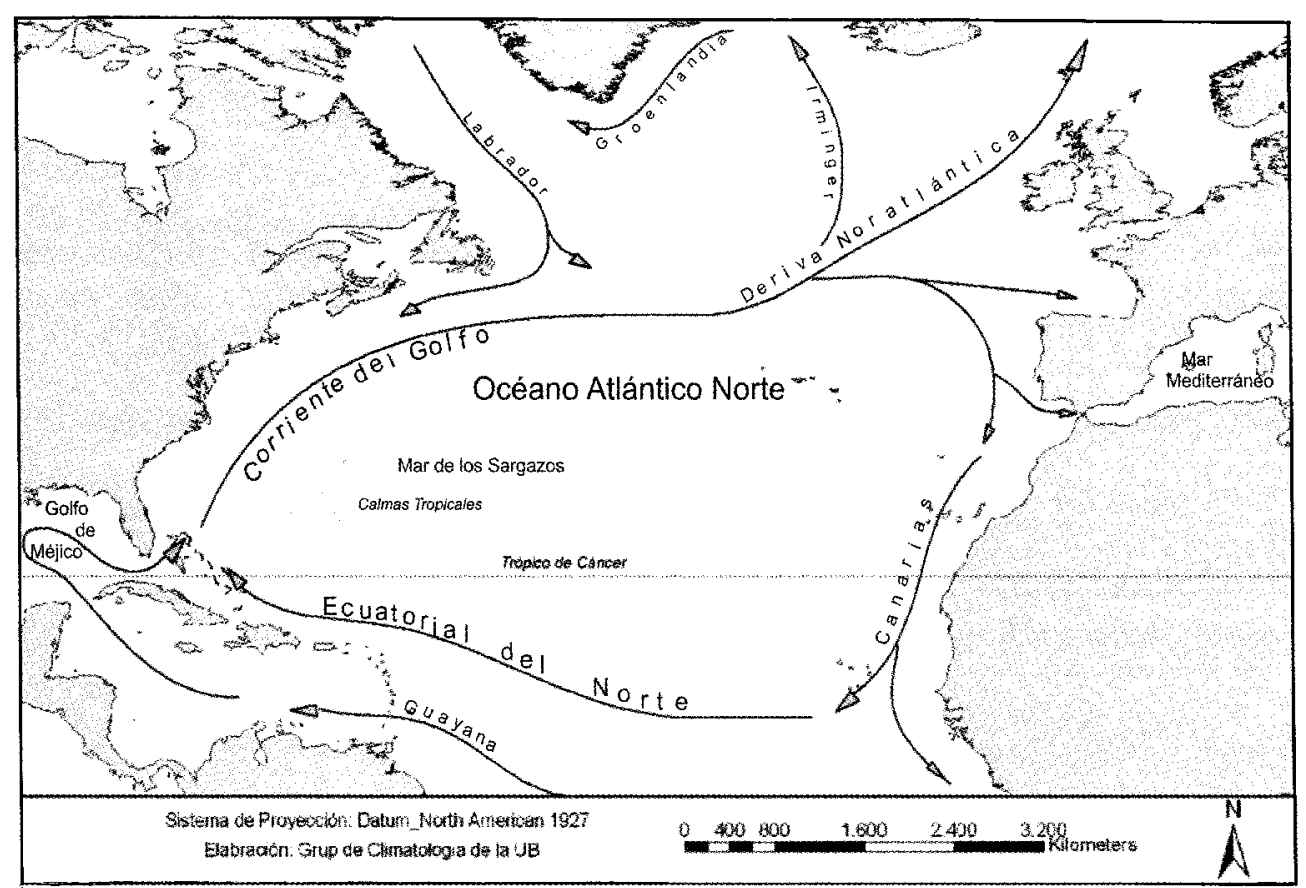

Figura 2. Esquema de las principales corrientes marinas del Atlántico Norte.

\section{Objetivos de la investigación y justificación del período de estudio 1939-1969}

En este estudio se pretende hallar una relación entre la pluviometría de la Península Ibérica y la actividad solar. En consecuencia, cabe definir un período de estudio que disponga de datos de precipitación a partir de registros instrumentales de una red, relativamente densa, coherente con el presente propósito de estudio, como es el siglo XX, y sobre todo, posterior a la Guerra Civil. Asimismo, hay que analizar un espacio temporal interesante desde el punto de vista de la actividad solar. Para estudiar la respuesta de estas anomalías térmicas marinas en la pluviometría de la Península Ibérica, se emplean los registros de precipitación a resolución anual con el fin de anular las variabilidades estacionales naturales (Perry, 1994).

Desde 1749, año desde que se disponen datos de manchas solares a resolución mensual, se enumeran los ciclos de 11 años de la actividad solar, denominados con el nombre de su descubridor, H. S. Schwabe. En la actualidad el ciclo número 23 se encuentra en su fase final, un ciclo moderadamente activo si es comparado con los ciclos 18 y 19 , o los 21 y 22. El primer par constituye el período de mayor actividad 
solar desde que existe dicha contabilización, ya que el ciclo 19 es el más activo de todo el registro desde 1749. En consecuencia, los ciclos 18 y 19 se seleccionan para el análisis. Éstos abarcan el período 1944-1964, con un promedio de longevidad inferior a los 11 años por ciclo, ya que los ciclos de mayor actividad se caracterizan por una menor longitud de onda, al contrario de lo que sucede con los de menor actividad. No obstante, 21 años es un período de estudio demasiado efímero para el análisis de un estudio de estas características, y en consecuencia, se ha pretendido ampliarlo hasta los máximos de los ciclos adyacentes, derivando el periodo en 31 años, 1939-1969. En la figura 3 se observa los distintos ciclos de las manchas solares contabilizados con el número de Wolf (ver apartado 3.1). Los ciclos 18 y 19 son en su conjunto el período más activo de todo el registro, aunque seguido de cerca por el espacio temporal comprendido por los ciclos 21 y 22.

Por tanto, se resume el objetivo del presente trabajo en analizar la posible presencia de una relación significativa entre los totales anuales de precipitación registrados en distintos observatorios instrumentales de la Península Ibérica en el período 1939-1969 y los ciclos solares 18 y 19.

Por último, cabe añadir que el tramo temporal 1939-1969 es posterior a un período de relativa baja actividad solar, que comprende desde finales del siglo XIX hasta mediados de los años 30 del siglo XX. Durante este curso de tiempo, según Goldberg (1978), se desvanece cualquier influencia solar sobre distintos elementos meteorológicos de varias áreas del globo, hecho que ya intuyó en su momento Lawrence (1965) cuando sugería que las señales solares eran más nítidas durante los períodos de máxima actividad solar.

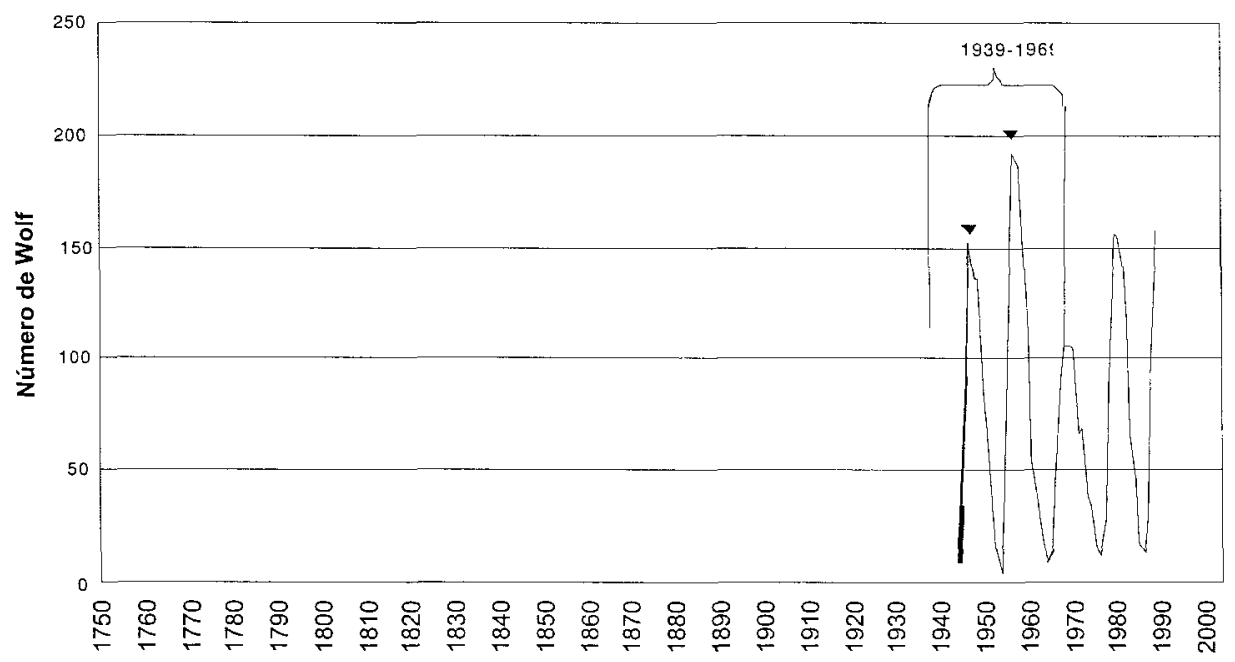

Figura 3. Evolución de las manchas solares mediante el índice del número de Wolf a resolución anual (1750-2003). 


\section{Datos y Metodología}

\subsection{Número de Wolf}

El flujo emitido por las fáculas ha sido medido desde la Tierra desde 1947, y se ha comprobado que sus fluctuaciones siguen el modelo de las variaciones de las mismas manchas solares (Lean y Foukal, 1988).

Esto permite justificar el hecho de que, para cuantificar la actividad solar en este estudio, se utilice el registro del número de Wolf anual disponible en la página web del SIDC-RWC Belgium World Data Center del Observatorio Real de Bélgica. El número de Wolf no es directamente el número de manchas presentes en la esfera solar, sino que responde a una definición del astrónomo suizo Johan Rudolph Wolf, que desarrolló con el propósito de dar objetividad a la medida y aproximarla más a un índice modelador de la actividad solar (Vázquez Abeledo, 1998):

$$
R=k(10 g+f)
$$

donde $\mathrm{g}$ es el número de grupos de manchas; $\mathrm{f}$, el número de manchas individuales; y k, la constante de calibración que tiene en cuenta las condiciones observacionales.

Actualmente, se tienen los datos y herramientas suficientes para poder diseñar un modelo adecuado que describa la actividad solar de forma rigurosa y exacta, pero la longitud de los datos disponibles mediante el índice del número de Wolf hace que éste siga siendo un referente para realizar estudios de carácter histórico.

\subsection{Series instrumentales de precipitación de la Península Ibèrica}

Se han empleado 35 series pluviométricas, 32 de estaciones españolas y 3 de portuguesas (Figura 4). Las series portuguesas provienen del Sistema Nacional de Informação de Recursos Hidricos de Portugal, mientras que el resto son del Instituto Nacional de Meteorología de España. Los datos de las series y su correspondiente homogeneización proceden del trabajo de Vicente-Serrano y Beguería Portugués (2004). Algunas series han sido creadas con los datos de diferentes observatorios de una misma localidad. Las series fueron comprobadas usando un control de calidad que identificó los registros anómalos con la ayuda del programa AnClim (_t_pánek, 2003). Para garantizar la calidad final de éstas, la homogeneidad de cada una se comprobó con el Standard Normal Homogeneity Test (SNHT) (Alexandersson y Moberg, 1997). Las pocas series no homogéneas identificadas se corrigieron y las lagunas temporales fueron completadas mediante regresiones lineales con las respectivas series de referencia. Así, queda asegurada la homogeneidad de las series y, en consecuencia, la calidad de los análisis. 
El espacio territorial de la Península Ibérica está cubierto en su totalidad, a excepción del cuadrante noroccidental, entre el norte de Portugal y Asturias. No obstante, las series del noroeste de Castilla, y de Galicia, son susceptibles de representar alguna correspondencia en el conjunto de la zona del noroeste peninsular.

\subsection{Tratamiento estadístico}

Se ha ensayado la existencia de una relación entre dos variables cuantitativas utilizando el método de la correlación. La cuantificación de la fuerza de la relación entre dos variables, en este caso la actividad solar representada por el número de Wolf, y la precipitación anual registrada en diferentes observatorios de la Península Ibérica, se estima mediante el cálculo del coeficiente de correlación de Pearson (r). Un coeficiente muy empleado en Climatología, sobre todo, para hallar relaciones entre variables climáticas. Un ejemplo representativo, son las correlaciones que se hacen entre la precipitación u otras variables y los patrones de teleconexión como la NAO (Ulbrich et al., 1999; Martín-Vide y Fernández, 2001; Trigo et al., 2004), la WeMO (Martín-Vide y Lopez-Bustins, 2006) o el ENSO (Knippertz et al., 2003; Rodó et al., 1997) en la presente área de estudio. De todas formas, es la metodología tradicional usada para hallar relación entre los ciclos solares y las variables climáticas (Hoyt y Schatten, 1997).

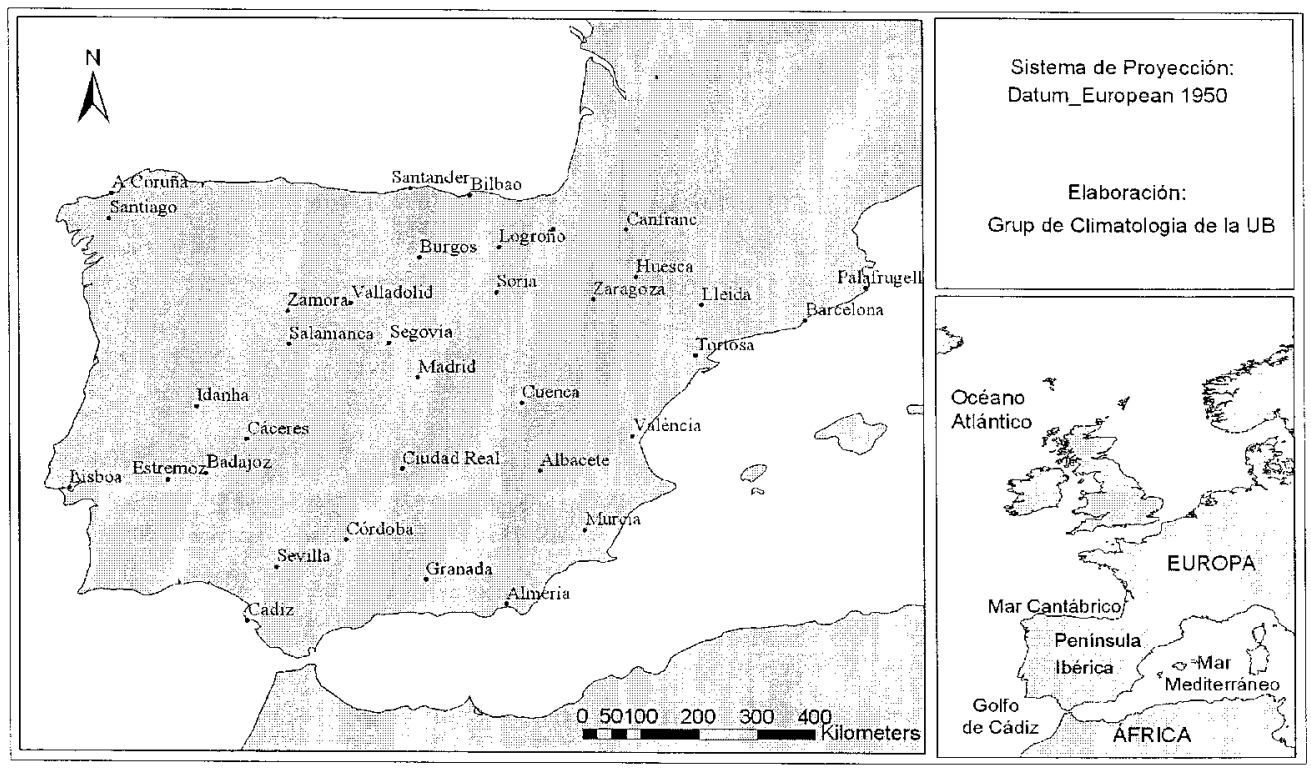

Figura 4. Localización de las series pluviométricas de estudio en el marco geográfico físico de la Península Ibérica. 
Se tiene plena conciencia que la correlación no implica causalidad. El hecho de que estén relacionadas dos variables no implica que una provoque las variaciones en la otra, esto último deberá justificarse de otra forma. La causalidad es un juicio de valor que requiere más información que un simple valor cuantitativo de un coeficiente de correlación, pero el cálculo de éste ya proporciona cierta información.

En definitiva, la metodología de análisis en este estudio es calcular los coeficientes de correlación de Pearson ( $r$ ), relacionando los datos de precipitación de cada una de las estaciones con el registro de manchas solares; asimismo, aplicando diferentes desfases temporales entre las dos series. En concreto, se analiza el caso de una reacción inmediata a los cambios de la actividad solar en la variabilidad de la precipitación, desfase de 0 años, hasta llegar a los 11 años de desfase temporal. Por tanto, se han empleado estas series pluviométricas hasta 1980 para no reducir el número de parejas correlacionadas en cada desfase. Se ha mantenido fijo el período 1939-1969 para el número de Wolf al ser la actividad solar la variable independiente

Una vez hallado $r$, se determina la significación de cada coeficiente calculado utilizando un test basado en la distribución de la T Student. Para ello se calcula el error estándar:

$$
e s=\sqrt{\frac{1-r^{2}}{n-2}}
$$

En nuestro caso $\mathrm{n}=31$. Con el valor de la $\mathrm{T}$ Student, se procede a calcular la $\mathrm{r}_{\text {limite: }}$ :

$$
\mathrm{r}_{\text {limite }}=\mathrm{es} \cdot \mathrm{t}_{-}(\mathrm{n})
$$

donde $\mathrm{t}$ (n) es un valor extraído de la tabla correspondiente a la distribución $\mathrm{T}$ Student y fundamentalmente su determinación depende de $n, y$ _ es un valor que depende del nivel de significación en el que se decida trabajar (95\% es el nivel de significación utilizado en Climatología, siendo $\_=0.05$ ) .

Si el valor absoluto de $r$ es superior o igual a $r_{\text {limite, }}$, se considera la posibilidad de que las dos variables estudiadas estén correlacionadas. Todos los $\mathrm{r}_{\text {límite }}$ de los coeficientes de cada serie se hallan en el intervalo (0.29-0.37). Esto es importante para entender la representación de los resultados en los mapas. En ellos se traza con una línea más gruesa la isolínea 0.30 , con la finalidad de delimitar esas zonas con una correlación significativa o próxima a la significación. Esta misma isolínea, pero de signo negativo, se ha dibujado con líneas discontinuas (Figura 5). 


\section{Resultados}

\subsection{Mapas de distribución de las regresiones pluviometría ibérica-número de Wolf (1939-1969)}

Si atendemos al mapa obtenido sin ningún desfase temporal ( 0 años) de la figura 5 , los coeficientes más altos de correlación indican qué zonas reaccionan con cierta inmediatez a los cambios de la actividad solar. Especialmente, se trata de la región situada en torno al golfo de Valencia, siendo el coeficiente más elevado el de la misma ciudad de Valencia. En concreto, se trata de una $r=0.63$. Los resultados también son significativos en los puntos de la Meseta que se encuentran bajo la influencia de la dinámica mediterránea, como es el caso de Albacete, con $r=0.42$. La configuración es semejante al área delimitada como ámbito estrictamente mediterráneo de la Península Ibérica por el Dr. Martín-Vide mediante la distribución espacial del indice de concentración de la precipitación diaria (Martin-Vide, 2004). Esta respuesta inmediata vendría justificada por la dependencia de su régimen pluviométrico de la ciclogénesis propia del Mediterráneo, y éste, al ser un mar de reducidas dimensiones y casi cerrado, responde rápidamente a cualquier anomalía térmica producida por el incremento o reducción de radiación solar durante el ciclo de Schwabe.

En esta primera distribución de los coeficientes sin desfase temporal, dentro del marco mediterráneo, se puede distinguir que las costas orientadas al sur son más dependientes de las advecciones asociadas a bajas atlánticas desprendidas hacia latitudes ibéricas y de otras situaciones atlánticas. Así es como se refleja en la costa Central catalana y en la mitad oriental de Andalucía al debilitarse esta correlación sin desfase. En consecuencia, son zonas en que la Oscilación del Mediterráneo Occidental pierde peso frente a la NAO (Martin-Vide y Lopez-Bustins, 2004). De este modo, por contraste, se puede apreciar que las costas orientadas al este son las que obtienen unos valores del coeficiente de correlación más elevados con este desfase temporal nulo.

En los resultados obtenidos con un año de desfase, se desdibuja el área del golfo de Valencia que aparecía tan bien definida en el mapa anterior. Con dos años de desfase temporal, este núcleo significativo alrededor de las costas de Valencia y Murcia desaparece totalmente, generándose, en cambio, la formación de una franja significativa que abarca casi todo el norte peninsular. Sobre todo es especialmente clara en las regiones bañadas por el golfo de Vizcaya. En el mapa correspondiente a 3 años de desfase, la zona del norte enmarcada con la isolínea de $r=0.30$ se ve aún más ampliada con ciudades como Pamplona1 con un coeficiente de correlación de $r=$ 0.52, Bilbao $(r=0.46)$, Santander $(r=0.37)$, Santiago de Compostela $(r=0.39)$, Logroño $(r=0.46)$ o Burgos $(r=0.37)$. En consecuencia, es el momento en que la señal solar alcanza una mayor extensión en el territorio peninsular, hipotéticamente vinculado a la llegada a las costas cantábricas de las anomalías térmicas marinas sub- 
superficiales transportadas por la corriente del Golfo. Esta área de máxima influencia se extiende en su límite meridional hasta los relieves del Sistema Central que separan las mesetas ibéricas. Sus contrafuertes más septentrionales, junto con los relieves centrales más elevados del Sistema Ibérico, actúan como de divisoria relativa entre el ámbito atlántico septentrional y el mediterráneo.

En este mismo mapa de desfase 3 años, también aparece un núcleo importante de coeficientes relativamente altos en el valle del Guadalquivir y proximidades. Los valores de correlación son importantes en ciudades como Sevilla, aunque éstos aún son más elevados en los resultados obtenidos con 4 años de desplazamiento temporal. En concreto, se trata de una $r=0.42$ para la ciudad mencionada. Se deduce que la deriva noratlántica, al noroeste peninsular, se bifurca entre un ramal hacia el mar Cantábrico, y otro, el más caudaloso y principal, que emprende una dirección sur hacia el golfo de Cádiz, hasta derivar en la corriente fría de las Canarias, como inicio del retorno que cierra la circulación del océano del Atlántico Norte. Por tanto, no es de extrañar un cierto retraso en la llegada de las anomalías térmicas marinas al marco suroeste peninsular. Asimismo, tampoco se espera que la anomalía llegue ahí con la misma intensidad, por tanto, no siempre alterará la precipitación; al hilo de ello se explica que los coeficientes no sean tan significativos (Sevilla $r=0.42$, Cádiz $r=0.34$, Estremoz $r=0.33$ y Córdoba $r=0.32$ ).

Resumiendo estos primeros mapas, las zonas influenciadas por las corrientes del Atlántico tienen dos tiempos de respuesta diferentes a la acción de la actividad solar. Los tiempos se rigen en relación con el período de desplazamiento de las aguas septentrionales a las suroccidentales peninsulares.

La distribución espacial de los coeficientes, transcurridos 5 años, es de signo negativo. En concreto, en toda el área del golfo de Valencia las isolíneas tienen la misma configuración que en el mapa de desfase temporal 0 años. La explicación a este fenómeno se halla en el hecho de que, al cabo de cinco años aproximadamente, se invierte la evolución de las manchas solares. Durante los primeros cinco años, la actividad va aumentando, así como el número de manchas, hasta que llega a un máximo. Después de éste, el ciclo se invierte y la actividad, junto con el número de manchas, va disminuyendo. La variación de la actividad solar, a partir de ese momento, va en sentido contrario, y en el mapa este fenómeno se refleja en una configuración de las isolíneas semejante a la del primer mapa sin desfase temporal, pero con signos contrarios. Asimismo, las anomalías negativas de las aguas marinas producidas durante los mínimos de los ciclos solares de 11 años, se transforman en déficits hídricos por una mayor sequedad del entorno atmosférico producido (Perry, 1994; Palmer y Brankovic, 1989). Por otro lado, las mayores anomalías negativas de precipitación en el golfo de Valencia se observan como respuesta a los mínimos solares al cabo de 5 años, período inferior a la mitad de un ciclo de Schwabe, atribuido a la disminución de la longevidad del ciclo solar cuando éste es especialmente activo, como es el caso de los ciclos 18 y 19.

Los mapas de los desfases siguientes no muestran ningún vínculo con la variabilidad de la actividad solar, así como tampoco se dibujan explícitamente relaciones nega- 
tivas para las regiones peninsulares atlánticas con un desfase temporal de 7-9 años. Éstas son susceptibles de analizarse más especificamente en el siguiente apartado.

\subsection{Evolución de los coeficientes de correlación en los distintos ámbitos climáticos de estudio}

En función de la zona de la Península Ibérica, los coeficientes de correlación ofrecen unos valores y una evolución concretos. En la figura 6 se analiza por áreas de influencia la evolución de los coeficientes de Pearson. Se seleccionan ciudades representativas de cada ámbito climático donde se detecta cierta relación. De este modo, se han definido tres áreas o ámbitos: El mediterráneo, que comprendería el arco este

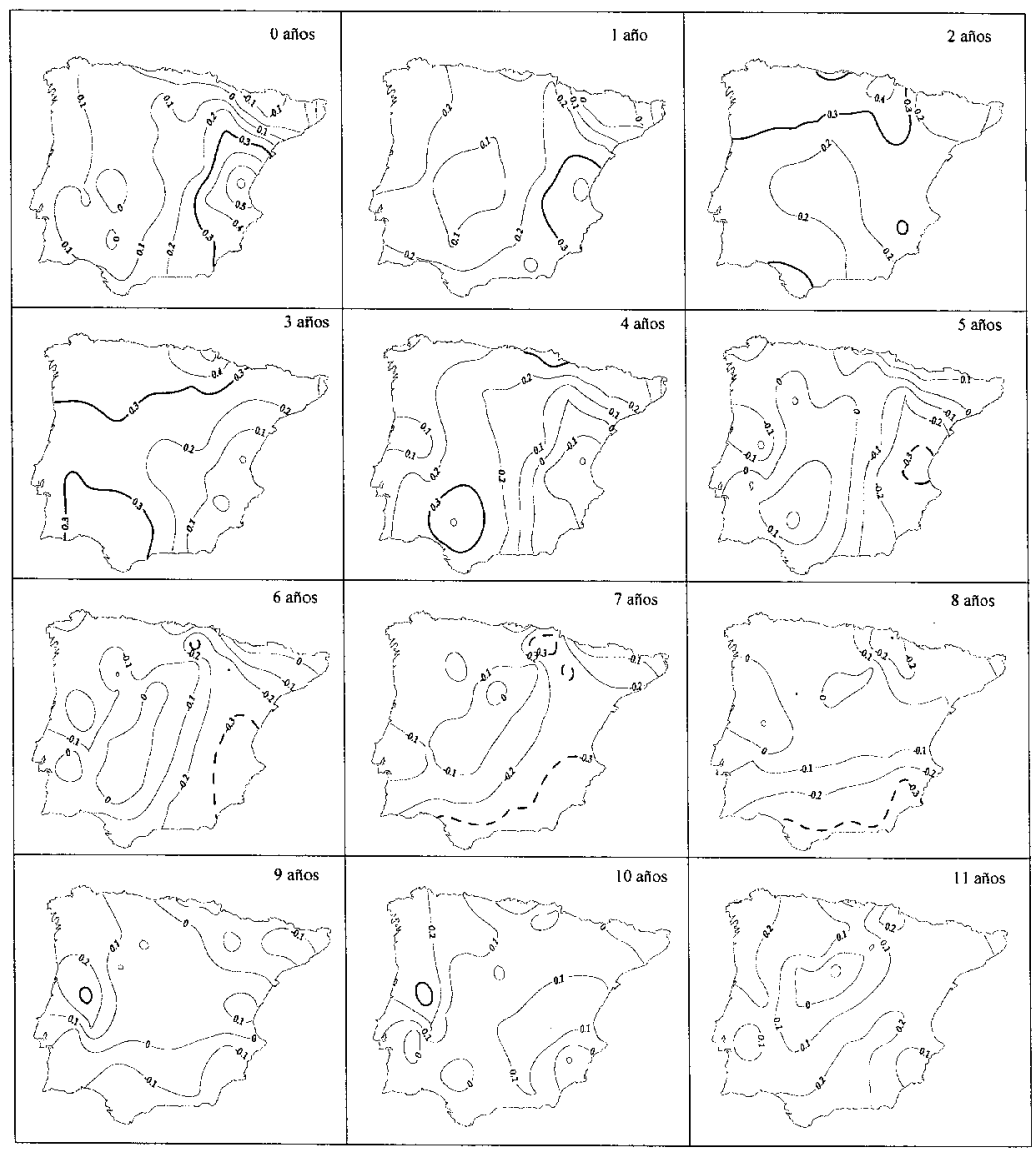

Figura 5. Distribución de los coeficientes de correlación entre las pluviometrías de los puntos de estudio y el número de Wolf a resolución anual con distintos desfases temporales (1939-1969). 
peninsular, el atlántico septentrional, con la totalidad de la cornisa cantábrica y alrededores, y el atlántico meridional, la cuenca del Guadalquivir y proximidades. Por otro lado, entendemos por ciudades representativas aquéllas que tienen un comportamiento pluviométrico típico del área definida.

La gráfica de la figura 6.a. corresponde a la evolución de 4 ciudades representativas del primer grupo, el ámbito mediterráneo. Su nivel máximo de correlación es a los 0 años de desplazamiento temporal. Con el paso del tiempo, la actividad solar deja de ejercer su influencia, y el valor del coeficiente va disminuyendo de forma casi paralela en todas las ciudades, hasta hacerse negativo trascurrido medio ciclo solar, exactamente, cuando éste llega a su mínimo. La evolución temporal de los coeficientes de las ciudades representativas del ámbito atlántico norte peninsular coincide en un pico a los 3 años de desfase temporal. A pesar del máximo coincidente, el conjunto de sus evoluciones son similares, alcanzando valores correlativos negativos en el intervalo de 6 y 8 años tardíos. Esta horquilla temporal es el período que se corresponde a los mínimos solares que suceden al cabo de 5 años en los ciclos de Schwabe efímeros con un retraso de 3 años (Figura 6.b.).

A partir de los resultados obtenidos en el apartado 4.1. se calcula que la corriente atlántica, considerando su velocidad media de $6-8 \mathrm{~km} /$ día, necesita poco menos de un año para alcanzar las costas suroccidentales de la península Ibérica, ámbito que se denomina en este estudio como atlántico meridional peninsular. En consecuencia, el máximo de correlación con las manchas solares en este ámbito ocurre a los 4 años, aunque no es descartado el hecho de una correlación satisfactoria a los 3 años, y unos negativos, un año más tarde que en el atlántico septentrional peninsular, 7-9 años (Figura 6.c.).

Si bien cabe decir, que la influencia de las anomalias marinas no tiene un intervalo de tiempo estrictamente definido, sino que es un fenómeno gradual, tal como se representa en las gráficas de la figura 6. La evolución de las correlaciones es paulatina con un orden acorde a los ciclos solares de Schwabe.

a

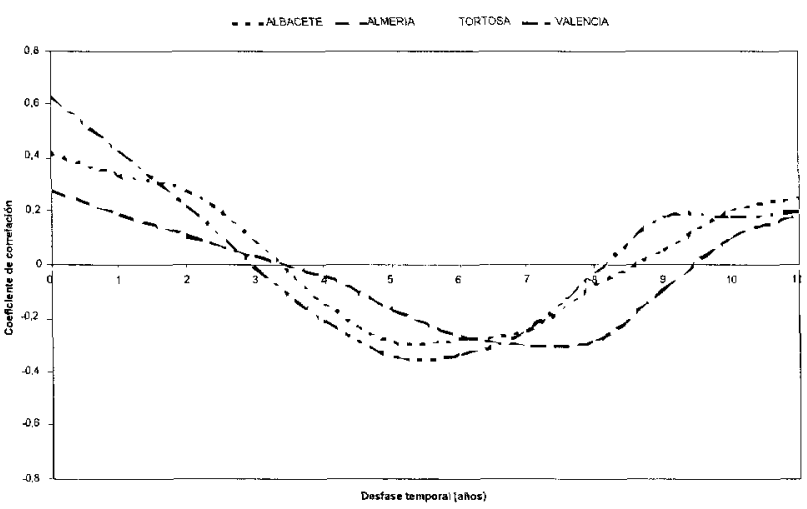

Figura 6. Distribución de los coeficientes de correlación entre las pluviometrías de los puntos de estudio y el número de Wolf a resolución anual con distintos desfases temporales (1939-1969). 
b

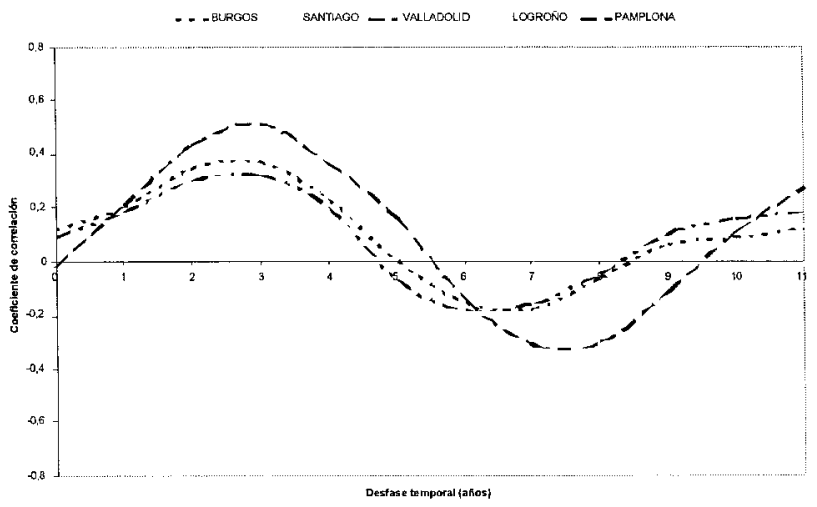

c



Figura 7. Evolución de los coeficientes para los distintos ámbitos peninsulares donde se halla correlación con la actividad solar: a. Mediterráneo. b. Atlántico Septentrional. c. Atlántico Meridional.

Para presentar resumidamente estas tres tendencias detectadas en el análisis, a continuación se seleccionan las tres series mejor correlacionadas de cada ámbito definido: Valencia, con una $\mathrm{r}=0.63$ a los 0 años, en el ámbito mediterráneo; Pamplona, con una $r=0.52$ a los 3 años en el atlántico septentrional, y Sevilla, con $r=0.42$ a los 4 años, como representativa del atlántico meridional.

Entre Pamplona y Sevilla se aprecia un sensible desfase de un año, que justifica en parte la posible hipótesis de la velocidad de la corriente atlántica en su descenso por las costas occidentales ibéricas. Sin embargo, Valencia tiene su máximo 3 y 4 años antes, respectivamente, que las series atlánticas. No obstante, la evolución de las curvas de correlación de las tres series son casi paralelas a pesar de ondularse distintamente en el tiempo (Figura 7) 


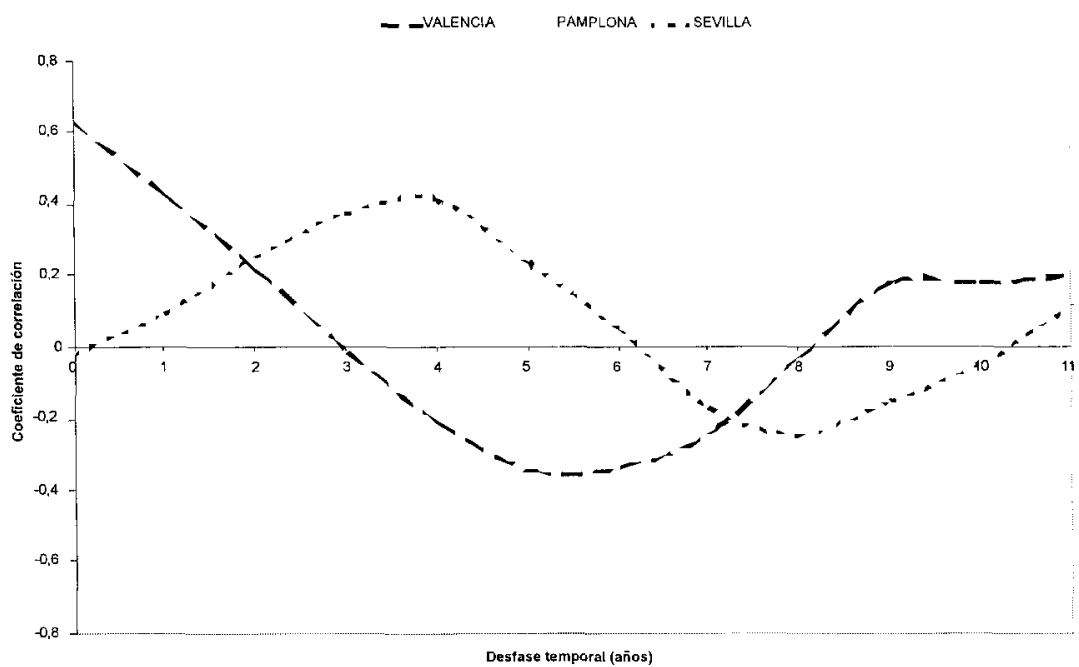

Figura 8. Evolución de los coeficientes de correlación de las tres series más representativas de cada ámbito de estudio definido.

\section{Discusión y conclusiones}

A lo largo de este artículo, se ha tenido presente que existe una gran dificultad en demostrar la influencia de la actividad solar en una variable climática en la troposfera. Y que a menudo, se puede concluir apresuradamente en hipótesis susceptibles de ser criticadas y contradichas. No obstante, la intención de este estudio es dar a conocer la existencia de estas correlaciones, que plantean la incertidumbre de una relación causal entre la pluviometría y la variación de la irradiancia solar a lo largo de los ciclos de Schwabe. Así, la corriente del Golfo, como partícipe del complejo mecanismo sol-océano-atmósfera, "conectaría" en este ensayo preliminar las citadas variables, para que posteriores análisis confirmen o rechacen la hipótesis inicial.

Los resultados más contundentes indican que la respuesta más alta en el ámbito peninsular de los ciclos 18 y 19 se produce en el golfo de Valencia, dada la configuración reducida y semicerrada del mar Mediterráneo, que permite una conexión simultánea. En la cornisa cantábrica, en especial las tierras que bañan el golfo de Vizcaya, la pluviometría responde con mayor señal a los 3 años, espacio temporal estimado para el transporte por parte de la corriente del Golfo de las anomalías térmicas marinas generadas en las áreas anticiclónicas tropicales del Atlántico Norte. Estas anomalías continúan su trayecto hasta el suroeste peninsular, para influenciar también en la precipitación de la cuenca del Guadalquivir al año siguiente. Las correlaciones no son muy altas, pero su distribución en el territorio y su evolución en el 
tiempo determinan una firme coherencia geográfica, la cual avala unos primeros resultados. De todas formas, la señal de la influencia solar en las series pluviométricas ya se esperaba, a priori, de carácter débil. En este sentido, se justifica el análisis llevado a cabo a resolución anual, ya que un estudio estacional o mensual propicia un aumento del ruido y la variabilidad; las influencias globales deben detectarse en un principio a una escala mayor, tanto temporal como espacialmente.

En futuros análisis se ampliará el período de estudio hasta los ciclos 21 y 22, así como se trabajará con datos de anomalías térmicas marinas de los distintos mares que rodean la Península Ibérica, con la finalidad de hallar en ellas ciertas ciclicidades acordes con los ciclos de Schwabe.

En definitiva, se ha presentado la variabilidad de la irradiancia solar como factor a añadir en el complejo grupo de variables que modulan la precipitación peninsular, mediante una vía de influencia directa, aunque, como se ha introducido, existen otras directrices en la influencia de esta variabilidad de la actividad solar en el clima. La aportación de este artículo puede estimular nuevos análisis que verifiquen o rechacen las hipótesis planteadas, que, a juicio de los autores, no han sido tratadas con tanto detalle como otras en el marco del tercer IPCC (Intergovernmental Panel on Climate Change), publicado en el 2001.

\section{Agradecimientos}

Agradecer la aportación y homogeneización de las series de precipitación por gentileza del Dr. Segio Vicente Serrano de la Universidad de Zaragoza. Asimismo, nos satisface haber contado con la supervisión de la interpretación de los resultados del Dr. Javer Martín Vide de la Universitat de Barcelona. Uno de los autores de este artículo, López-Bustins, disfruta de una beca predoctoral FPU del Ministerio de Educación y Ciencia. Las investigaciones de este artículo se han llevado a cabo en el seno del Grup de Climatologia (2005SGR-01034, Generalitat de Catalunya) (www.ub.edu/gc/menu.htm) de la Universitat de Barcelona y del proyecto IPIBEX CGL2005-07664-C02-01/CLI. Por último, agradecer a dos revisores anónimos sus respectivas sugerencias que han permitido mejorar, sobre todo, la estructura del trabajo.

\section{Bibliografía}

Alexandersson, H., Moberg, A. (1997): Homogenization of Swedish Temperature Data. Part I: Homogeneity Test for Linear Trends. International Journal of Climatology, 17, p. 25-34.

Baldwin, M.P., Dunkerton, T.J. (1999):
Propagation of the Arctic Oscillation from the stratosphere to the stratosphere. Journal of Geophysical Research, 104 (24), p. 30937-30946.

Baldwin, M.P., Dunkerton, T.J. (2005): The solar cycle and stratsphere-troposphere 
dynamical coupling. Journal of Atmospheric and Solar-Terrestrial Pbysics, 67, p. 71-82.

Benito, G., Díez-Herrero, A., Fernández de Villalta, M. (2003): Magnitude and Frequency of Flooding in the Tagus Basin (Central Spain) over the Last Millenium. Climatic Change, 58, p. 171-192.

Burroughs, W.J. (1992): Weather Cycles: Real or Imaginary?. Cambridge University Press, $201 \mathrm{pp}$.

Herman, J.R., Goldberg, R. A. (1978): Sun, Weatber and Climate. NASA SP-426, GPO, Washington, D.C., $360 \mathrm{pp}$.

Hoyt, D.V., Schatten, K.H. (1997): The Role of the Sun in Climate Change. New York: Oxford University Press, 279 pp.

IPCC Third Assessment Report: Climate Change uww ipcc.ch/pub/reports. btm.

Kirov, B., Georgieva, K. (2002): Long Term Variations and Interrelations of ENSO, NAO and Solar Activity. Pbysics and Chemistry of the Earth, 27, p. 441-448.

Knippertz, P., Ulbrich, U., Marques, F., CorteReal, J. (2003): Decadal Changes in the link between El Niño and springtime North Atlantic Oscillation and EuropeanNorth African Rainfall. International Journal of Climatology, 23, p. 1293-1311.

Labitzke, K., van Loon, H. (1999): The Stratospbere: Phenomena, History and Relevance, Springer, Berlin Heidelberg, New York. 179 pp.

Landscheidt, T. (1995): Global Warming or Little Ice Age? In: Finkl, CW, ed.: Holocene cycles. A jubilee volume in celebration of the $80^{\text {th }}$ birthday of Rhodes W. Fairbridge. Fort Lauderdale, The Coastal Education and Research Foundation (CERF), p. 371382.

Landscheidt, T. (2000): Solar forcing of El Niño and La Niña. In: Vázquez $\mathrm{M}$ and Schmieder B, ed.: The solar cycle and terrestrial climate. European Space Agency, Special Publication 463, p. $135-$ 140.

Lawrence, E.N. (1965): Terrestrial climate and the solar cycle. Weatber, 20, p. 334-343.

Lean, J, Foukal, P. (1988): A model of solar luminosity modulation by magnetic activity between 1954 and 1984. Science, 240, p. 906-908.

Lewis, M.R., Carr, M., Feldman, G.C., Esaias, W., McClain, C. (1990): Influence of penetrating solar radiation on the heat budget of the equatorial Pacific Ocean. Nature, 347, p. 543-545.

Liov, K.N. (1980): An Introduction to Atmospheric Radiation, Academic Press, New York, 392 pp.

Lucio, P.S. (2005): Learning with solar activity influence on Portugal's rainfall: A stochastic overview. Geophysical Research Letters, 32, L23819, doi:10.1029/2005GL023787.

Martín-Vide, J. (2004): Spatial distribution of a daily precipitation concentration index in peninsular Spain. International Journal of Climatology, 24, p. 259-971.

Martín-Vide, J., Fernández, D. (2001): El índice NAO y la precipitación mensual en la España peninsular. Investigaciones Geogrâficas, 26, p. 41-58.

Martín-Vide, J., López-Bustins, J.A. (2004): The Western Mediterranean Oscillation (WeMO) and its influence in the Eastern Spanish rainfall. A methodological approach. In $4^{\text {th }}$ EMS Annual Meeting Abstracts, vol. 1, poster 0166 .

Martín-Vide, J., López-Bustins, J.A. (2006): The Western Mediterranean Oscillation and Rainfall in the Iberian Peninsula. International Journal of Climatology, en prensa.

Montón Chiva, E., Quereda Sala, J. (1997): ¿Hacia un cambio climático?. La evolución del clima mediterráneo desde el siglo 
XIX. Castellón: Fundación DavalosFletcher.

Pallé, E., Butler, C.J., O'Brien, K. (2004): The possible connection between ionitzation in the atmosphere by cosmic rays and low levels clouds. Joumal of Atmospheric and Solar-Terrestrial Pbysics, 66, p. 1779-1790.

Palmer, T.N., Brankovic, C. (1989): The 1988 U.S. drought linked to anomalous sea surface temperature. Nature, 338, p. 54-57.

Perry, C.A. (1992): A correlation between precipitation in the Western United States and solar-irradiance variations. Proceedings of the American Water Resources Association Conference, Managing Water Resources During Climate Change, Reno, NV, 1-6, 3 , p. 990-998.

Perry, C.A. (1994): Solar-irradiance variations and regional precipitation fluctuations in the western USA. International Journal of Climatology, 14, p. 969-983.

Reid, G.C. (1991): Solar Total Irradiance Variations and the Global Sea Surface Temperature Record. Journal of Geopbysical Research, 96, p. 2835-2844.

Reid, G.C. (2000): Solar Variability and the earth's climate: Introduction and overview. Space Science Reviews, 94, p. 1-11.

Rodó, X., Baert, E., Comin, F.A. (1997): Variations in seasonal rainfall in Southern Europe during the present century: relationships with the North Atlantic Oscillation and the El Niño-Southern Oscillation. Climate Dynamics, 13, p. 275284.

SIDC-RWC Belgium World Data Center for the Sunspot Index. http://sidc.oma.be

_t_pánek, P. (2003): Anclim-software for time series analysis. Dept. of Geography, Fac. of Natural Sciences, MU, Brno.

Trigo, R.M., Pozo-Vázquez, D., Osborn, T.J., Castro-Díez, Y., Gámiz-Fortis, S., EstebanParra, M.J. (2004): North Atlantic Oscillation influence on precipitation, river flow and water resources in the Iberian Peninsula. International Joumal of Climatology, 24, p. 925-944.

Ulbrich, U., Christoph, M., Pinto, J.G., CorteReal, J. (1999): Dependence of winter precipitation over Portugal on NAO and baroclinic wave activity. International Journal of Climatology, 19, p. 379-390.

Vaquero, J.M., Gallego, M.C., García, J.A. (2002): A 250-year Cycle in Naked-eye Observations of Sunspots. Geopbysical Research Letters, 29 (20), 1997, doi:10.1029/2002GL014782.

Vaquero, J.M. (2004): Solar signal in the number of floods recorded for the Tagus river basin over the last millennium. Climatic Change, 66, p. 23-26.

Vázquez Abeledo, M. (1998): La Historia del Sol y el Cambio Climático. Madrid: McGraw-Hill, 488 pp.

Vicente-Serrano, S.M., Beguería Portugués, S. (2004): Analysis of the spatial patterns of drought magnitude in the Iberian Peninsula by means of the Standardized Precipitation Index (SPI) and the Generalized Pareto distribution. In EMS Annual Meeting Abstracts, vol. 1, 00175.

Wallace, J.M., Smith, C., Jiang Q. (1990): Spatial patterns of atmosphere-ocean interaction in the northern winter. Journal of Climate3, p. 990-998.

Willson, R.C., Hudson, H.S. (1988): Solar luminosity variations in solar cycle 21 . Nature, 322 , p. $810-812$.

\footnotetext{
1 Pamplona y Logroño son ciudades que se incluyen en el ámbito del atlántico septentrional del estudio aun estando en una cuenca que vierte al Mediterráneo. El análisis de sus pluviometrías con patrones de varjabilidad de baja frecuencia muestra un incremento de precipitación en ellas con advecciones de noroeste, y una disminución con advecciones de sureste (Martín-Vide y López-Bustins, 2006).
} 